\title{
The Cuban Health Sector \& Disaster Mitigation
}

\section{By Guillermo Mesa, MD, MPhil}

Over the past few years, the most important disasters affecting Cuba have caused minimal loss of human life: suffice to note that just 28 lives were lost in the 13 hurricanes and tropical storms that hit the country from 2001 to 2007 . But this was not always the case. In 1963, over 1,200 people died in Hurricane Flora alone.[1]

Today, Cuba is still a developing country facing serious problems due to limited resources. Yet, it differs in some substantial ways from many other poor nations: adult literacy in Cuba is 99.8\%,[2] schooling is compulsory through the 9th grade, and higher education is readily accessible and free. These factors have important implications for public awareness, participation and organization when it comes to disaster management.

In the case of health, a single universal public system provides primary through tertiary care free of charge, through some 14,000 neighborhood family doctor-and-nurse offices, 498 community polyclinics, 243 hospitals, and other specialized clinical, laboratory, rehabilitation and research facilities.[3] Senior homes, facilities for disabled persons, maternity homes, dental clinics, pharmacies, and optometry and other services are distributed nationwide. The system also includes a national telecommunications network and portal, hygiene and epidemiology centers, libraries and 22 medical sciences faculties. Nearly 490,000 people staff the health system, $69.4 \%$ of them women.[3]

Since the 2005 Hyogo Framework for Action was developed, Cuba and other nations have intensified efforts to reduce vulnerability to hazards - mitigation of disaster's potential impact. In Cuba's case, the main threats faced by the country are the periodic hurricanes, tropical storms and flooding that are becoming ever more common throughout the Caribbean.

The Cuban health system plays a pivotal role in the country's national disaster planning, preparation, response and recovery. It is part of a comprehensive effort coordinated at all levels by the national Civil Defense (see You Can't Stop the Rain this issue), which has evolved over the past 45 years. Its essential roles are:

1. Training health professionals in disaster medicine

2. Participation in community education efforts

3. Maintaining public health epidemiological surveillance

4. Providing disaster planning leadership for specific types of disasters and technical expertise for territorial risk reduction studies

5. Generating risk reduction studies within and for its own units

6. Planning and implementing medical services during disaster response and recovery

7. Planning and providing specialized medical attention during disasters (medical teams and other resources for vulnerable areas, care for persons with special needs, etc.)

\section{Training Health Professionals}

Since the 2004-2005 academic year, civil defense training received by Cuban medical students has included disaster medi- cine, taught during the 3rd, 4th, and 5th years of their six-year program.[4] Post-graduate courses are also available to physicians and other health professionals, including nurses, psychologists and specialists in information and communications technologies. A Master's Degree program is in final stages of approval.

Additionally, the Latin American Medical School curriculum offers 120 hours of disaster medicine to medical students studying in Cuba from the Americas, Africa and Asia. By July of 2007, 5,360 students from 32 countries had completed this course upon graduation.[4]

\section{General Themes for Disaster Medicine Academic Courses}

1. General aspects: concepts, definitions, fundamentals

2. Socio-economic aspects as disaster determinants

3. Disasters and interruption of health-disease equilibrium

4. Role of the community in confronting disasters

5. First responders for massive reception of the injured and ill, life-saving

6. Health sector preparedness for emergencies and disasters

7. Covenants and international humanitarian law; the International Red Cross

8. Management of mass cadavers in disaster situations

9. Plans for managing different types of threats or hazards

10. The environment and disasters

11. Epidemiological surveillance and disasters

12. Organization of medical care and activities of the Integrated System for Medical Emergencies (SIUM) in disaster situations

Source: Bello, B. La medicina de desastres: su inclusión en la formación del médico general integral. Power point. Enrique Cabrera Faculty of Medical Sciences, Havana, December, 2007.

The heads of Cuban medical teams serving abroad, and all members of the Henry Reeve Team of Medical Specialists in Disasters \& Epidemics, also receive special training on a periodic basis to update their knowledge and skills, carried out through the Disaster Medicine Departments at each of Cuba's 22 medical sciences faculties. (See Cuba's Man in Sichuan, China and Cuban Health Cooperation Turns 45, this issue).

Responsible for designing these training programs for health professionals is a national academic cluster, including nine PhDs and 15 Master's Degree-level professors, headquartered at the Latin American Center for Disaster Medicine (CLAMED). The Center also acts as the national information gathering and management hub for the health system's disaster preparation and response.

\section{Participation in Community Education Efforts}

With assistance from the Pan American Health Organization (PAHO), UNICEF and other international agencies, CLAMED has expanded its portfolio to include provincial disaster preparedness 


\section{UNDP \\ Caribbean Risk Management Initiative}

Formally launched in 2004, the Caribbean Risk Management Initiative (CRMI) has dual headquarters in Cuba and Barbados and is designed to build capacity within the Caribbean region to adequately address the growing occurrence of natural hazards and environmental risks, as well as to emphasize the concept of climate change and social vulnerability. CRMI's main work focuses on building human capacities, tools and networks in the region, highlighting best practices in various countries and strengthening cooperation among governments.

Program Manager Karen Bernard told MEDICC Review: "Eighty percent of the Caribbean population lives within one kilometer of the coast, so vulnerability is high. The point is to share experiences and technologies. Other Caribbean countries are anxious to learn from Cuba in terms of protection of human life in the face of hurricanes. Regional facilities are also important for these small countries: not all have the capability to set up hurricane tracking systems or models of prediction, another reason why Cuba's input and databases are key."

www.onu.org.cu/crmi

\section{CLAMED}

\section{Latin American Center for Disaster Medicine}

CLAMED, under the umbrella of Cuba's Ministry of Public Health, was founded in 1996 and is charged with collecting, organizing and sharing the latest national and international information on disaster medicine; multidisciplinary research in the field; and development of human resources and social participation in the face of disasters. The focus of CLAMED's work is national and regional, aimed at encouraging multilateral cooperation and risk reduction programs. CLAMED belongs to the Caribbean Disaster Information Network (CARDIN), headquartered at the University of the West Indies, Jamaica, a four-language database available at wwwcardin.wwimona.edu.jm:1104.

www.sld.cu/sitios/desastres and www.sld.culinstituciones/clamed/

\section{PRECIS Caribe}

\section{A Project of the Cuban Meteorology Institute (INSMET)}

PRECIS Caribe is an interactive website with results of the PRECIS Regional Climate Model applications for Central America, Mexico and the Caribbean. The site facilitates online access to climate change scenarios developed by the Cuban Meteorology Institute, using PRECIS (Providing Regional Climates for Impact Studies), a model developed by the Handley Centre of the UK's Meteorological Office. PRECIS Caribe is the Institute's contribution to regional efforts to evaluate impact of climate change and develop adaptive measures, and is supported by UNDP and Canada's Institute for Development Assistance (CIDA). precis.insmet.cu/eng/Precis-Caribe.htm workshops around the country, to deepen understanding of various issues, such as psychological support during disasters; effectiveness of risk reduction plans for hydro-meteorological and seismic disasters; preparing health personnel for seismic events; veterinary health in disaster situations; community preparedness for sudden disasters; and disaster mitigation, preparedness and response related to hazardous toxic substances.

CLAMED and the Cuban Red Cross also collaborate on a series of educational efforts for community volunteers and school children; and the health system actively participates in the annual Meteoro Disaster Preparedness and Response Exercise organized by Civil Defense, to test local and national disaster readiness.

It should be emphasized that public education and awareness is an important role for health care providers throughout the system, beginning with family physicians and nurses. This is part of a larger effort coordinated by Civil Defense, which involves schools, work centers, mass media, and social organizations, among others.

\section{Maintaining Public Health Epidemiological Surveillance}

The health system maintains epidemiological surveillance through the Hygiene and Epidemiology Centers located in each province and municipality. Family physicians and nurses, community polyclinics, and hospitals regularly report to health and Civil Defense authorities, contributing data to the health system's own "early warning system" - the national Health Trends Analysis Unit (UATS). (See Gorry C. Training an Eye on Epidemics: Cuba's National Health Surveillance System. MEDICC Review. $2005 ;(7) 7: 6-7$.) This role extends to the country's borders, working closely with Cuban Customs authorities.

\section{Providing Disaster Planning Leadership for Specific Types of Disasters}

According to national Civil Defense protocols, the health system bears primary responsibility for steering preparation and response for certain types of disasters, such as vector-borne and other epidemics. In these cases, specialized personnel in the Ministry of Public Health develop technical norms and specify key activities for disaster management, which in turn serve as guides for national and local institutions, provinces and territories as they generate the required risk reduction studies (see You Can't Stop the Rain, this issue).

In addition, throughout the disaster management cycle, health directors at all levels - provincial, municipal, institutional - serve as the highest health sector Civil Defense authorities. They also represent the 
health sector in local government commissions that prepare risk reduction studies, drawing on specialists and population health experts.

\section{Risk Reduction Studies Within Its Own Units}

More rigorous risk reduction studies are mandated annually by Civil Defense Directive \#1 issued in 2005, applied to every institution in the country, including hospitals and polyclinics. Each center is required to evaluate its vulnerability to hazards, and proceed with an action plan to reduce them, which is incorporated into its yearly budget. Of course this is particularly important for the health sector, since keeping the hospitals and clinics running is essential to effective disaster response. Some vulnerabilities have been reduced across the board in the last four years, with installation of electric generators in the country's polyclinics, hospitals and other health care facilities.

Over 250 of the country's 498 community polyclinics and a number of older hospitals have been refurbished in the same period. Others are in process of remodeling. However, this does not automatically offer sufficient protection: some installations, for example, are located in particularly vulnerable spots - such as the America Arias Maternity Hospital in Havana, just a few blocks from the shoreline.

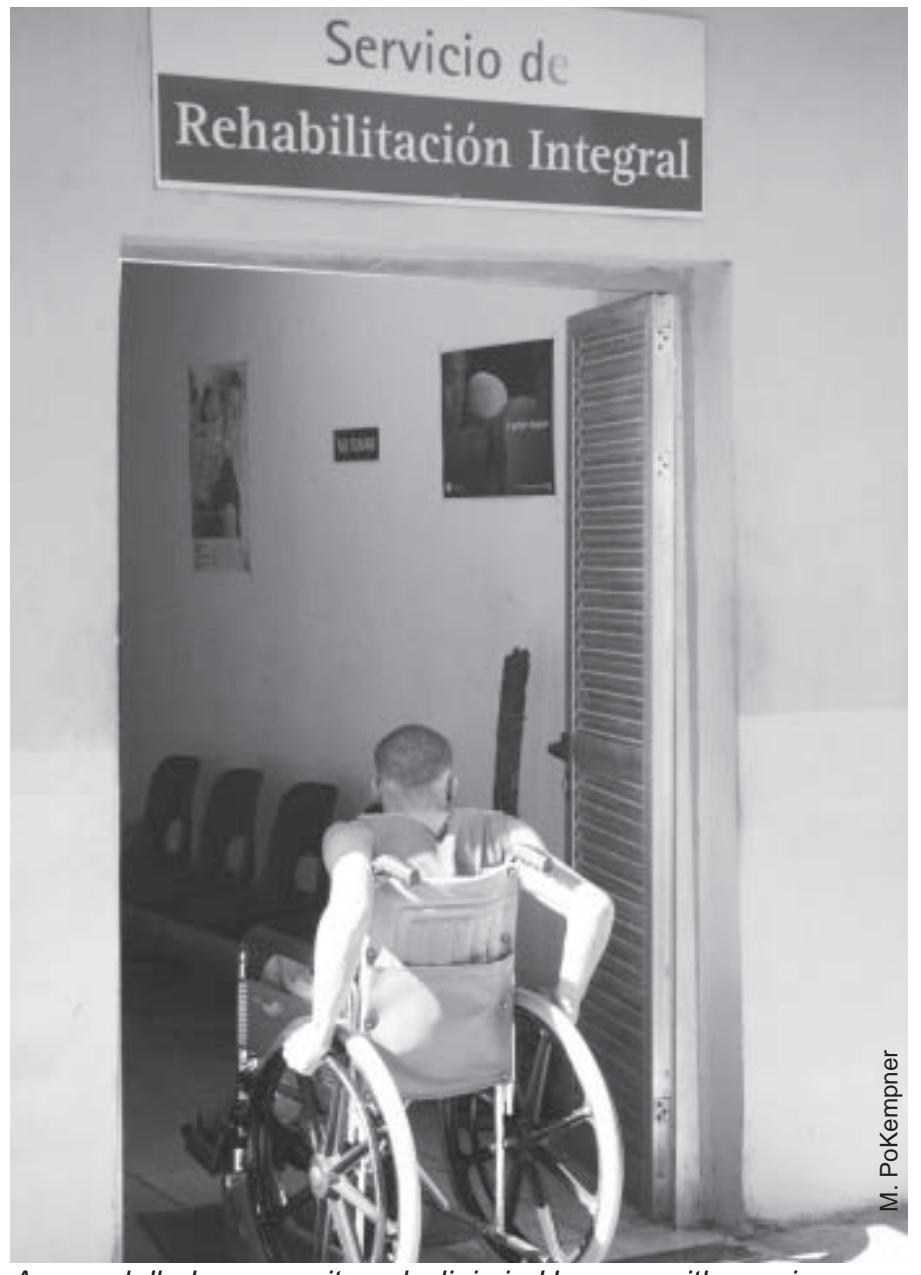

A remodelled community polyclinic in Havana: with repairs came new services such as physical therapy and rehabilitation.
Directive \#1 has also imposed requirements on the building of new health facilities, the blueprints and location of which require approval by disaster experts to minimize vulnerability to catastrophes.

Cuba is participating in an important initiative taken this year, the "Hospitals Safe from Disasters" campaign led by the World Health Organization and the UN's secretariat of the International Strategy for Disaster Reduction (ISDR). Noting that damage or destruction of health facilities is a major problem in disaster situations, the goal of the "safe hospitals" movement is to incorporate mitigation measures aimed at keeping the services open when they are most needed.[5]

PAHO and Cuban health authorities have jointly established specific indicators for determining not only safe hospitals, but also safe polyclinics, family physician offices, and other medical care facilities. Six hospitals have begun the evaluation process, which includes a detailed review of their location, structural conditions, patient flow during disasters, resources (water, electricity, communications, waste disposal), and stockpiles (medicines, water, food and others), among other aspects.

\section{Planning and Implementing Medical Services}

These plans form part of the comprehensive disaster plan at each territorial level, including risk reduction (mitigation), preparedness, response and recovery. In the case of public health, the planning methodology can be summarized as follows: $[6,7]$

- Risk assessment.

- Mitigation measures (reducing vulnerabilities, as above).

- Disaster response measures (preparedness, during disaster, recovery), including:

- Organization of preventive medical care;

- Organization of hygiene and epidemiology measures

- Evacuation of sick and injured (plus health inspection and certification of all large shelters, including their food preparation facilities) ;

- Logistics (health personnel by category for each center; hospital beds available for different types of disasters; minimum medications required for a given period and storage facilities; reserves of disinfectants and other key supplies; sources of water, gas, medicinal oxygen, etc.; ambulances and transportation);

- Organization of management and communications (lines of command and communication, alternative communications);

- Education of the general public and health personnel; and

- Cooperation with other sectors.

- Territorial mapping of health units, evacuation shelters, locations to be reinforced with medical teams, high-risk zones for the population, warehouses, water supply sources, etc.

- As appendices: warning plan, plan for each phase, community-level risk maps, agreements with other sectors (transportation, communications, etc.), and norms for reporting damages. 


\section{Policy \& Practice}

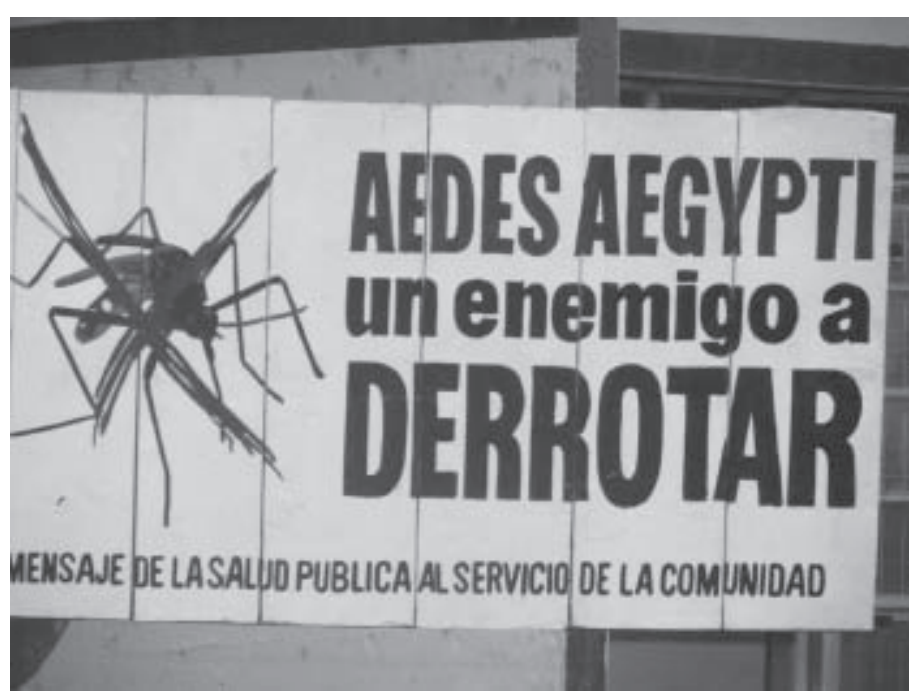

Public health billboard during campaign against dengue carrier mosquito Aedes aegypti.

These plans also include activation of the Integrated Medical Emergency System (SIUM), first responders in times of disaster. Under normal conditions, the SIUM's life-saving and rescue operations provide transportation and vital support for individual patients until they can be stabilized in polyclinic or hospital emergency services. Like all institutions in the health system, SIUM prepares on the municipal, provincial and national level for disasters involving massive victims.

In the immediate recovery phase, healthcare facilities evaluate the extent of their human and material losses, and take measures to re-establish services where they have been interrupted, beginning with the most essential. The health system's role in hygiene and epidemiological surveillance is particularly important in this phase - including testing the quality of water and preventing introduction vector-borne diseases. For example, in 2005, when Hurricane Wilma resulted in flooding along Havana's shoreline, public health teams - including hundreds of medical students - went house-to-house to test for water contamination.

Planning and Providing Specialized Medical Attention While this aspect is covered in the plans referred to above, it is worth emphasizing, since specific planning is required to ensure medical care for certain populations, such as pregnant women, the sick, small children, and the disabled, as well as psychological attention for persons affected by disasters. For areas apt to become isolated in a disaster, planning includes maintaining reserves of water, food and medications in the communities themselves. Medical-surgical teams are assigned to each one, with the necessary resources to function.

In the face of disasters to come, challenges for the Cuban health system include better planning for simultaneous catastrophes. Given the country's economic constraints, we are also challenged to fulfill the Directive \#1 goal of reducing vulnerabilities to a minimum in our own institutions, meriting accreditation as health care units "safe from disasters". Finally, more work needs to be done to develop a solid "culture of safety" in the public at large and also among health workers. In the end, the results of this education will do the most to ensure that reduction of vulnerabilities becomes a permanent course of action. 1 -

\section{References}

1. Civil Defense High Command. La reducción de desastres y los ciclones tropicales en Cuba. Defensa Civil de Cuba. 2006; (1):5-10.

2. PNUD. Informe sobre desarrollo humano 2007-2008. New York. 2007

3. Ministerio de Salud Pública. Anuario Estadístico de Salud 2006. Havana, 2007

4. Bello, B. La medicina de desastres: su inclusión en la formación del médico general integral. Power point. Enrique Cabrera Faculty of Medical Sciences, Havana, December, 2007

5. International Strategy for Disaster Reduction. Press Release: Campaign launched on hospitals safe from disasters. UN/ISDR 2008/2. 24Jan08. WHO, Geneva.

6. Conde H, García V. Preparación del sector salud para casos de emergencias y desastres. In: Gerencia de Desastres en Cuba. 2003. Red Caribeña para Información sobre Desastres (CARDIN). University of the West Indies, Jamaica. p.38-41.

7. Mesa, G. Estructura del plan contra desastres. Power point presentation. CLAMED, Havana. 2008. 